\title{
STUDY OF MODIFIED TBA DRIVER SCHEME*
}

\author{
A.V. Elzhov, A.A. Kaminsky, A.K. Kaminsky, V.I. Kazacha, E.M. Laziev, E.A.Perelstein, \\ S.N. Sedykh, A.P. Sergeev, Joint Institute for Nuclear Research, 141980, Dubna, Russia
}

\begin{abstract}
A modified scheme of two beam accelerator (TBA) [1] driver based on a linear induction accelerator is studied in this paper. In the proposed scheme the electron beam bunching occurs at a rather low initial energy $\sim 1 \mathrm{MeV}$. The bunched beam is accelerated in the accompanying enhanced synchronous microwave that provides the steady longitudinal beam bunching along the whole driver. There is no total microwave power extraction anywhere in the driver. A waveguide (initially corrugated and regular further) is used along the driver [2]. The simulation showed that a steady state is achieved when electron bunches accompanied after a TWT by an amplified microwave are simultaneously accelerated in an external electric field both for continuously distributed system parameters and for discrete parameters of accelerating sections. In the steady state the whole power, which is inserted into the beam by the accelerating field, transforms into the microwave power. Such kind of systems can serve effectively as a rather long (hundreds of meters) driver for the TBA concept. The experimental setup based on the JINR LIA-3000 facility to test the new scheme is under construction at present. It consists of the $800 \mathrm{keV}, 200 \mathrm{~A}$ injector and two reaccelerating sections. The first experimental results of the beam bunching study have been presented.
\end{abstract}

\section{INTRODUCTION}

Some schemes of the TBA driver based on a linear induction accelerator (LIA) were discussed in [1-5]. In these schemes the driver electron beam moves through an alternating discrete row of microwave generators (free electron lasers (FEL), relativistic klystrons, etc.) and reaccelerating sections. The microwave power is totally extracted from the driver after every generation section. Such kind of driver was experimentally investigated in [6], where the reacceleration of a modulated with the chopper help electron beam through two induction accelerator cells was demonstrated. The continuous extraction along the whole driver length of the microwave power was designed in CLIC [7] and a corresponding beam dynamics was studied also in [8].

A new scheme of two beam accelerator (TBA) driver based on a linear induction accelerator is suggested in this work. The scheme is quite uniform and has the following characteristic properties: a) the electron beam bunching occurs at a rather low $\sim 1 \mathrm{MeV}$ initial energy; b) the bunched beam further acceleration occurs in the accompanying enhanced microwave that provides the steady longitudinal beam bunching along the whole driver; c) there is no total microwave power extraction anywhere in the driver; d) a waveguide is used along the driver.

The driver consists of an injector, buncher and long (a few hundreds of meters) row of separate LIA sections producing the external accelerating electric field and partitioned by transition chambers. The injector produces the initial electron beam with energy 1 through $2 \mathrm{MeV}$ and current 0.5 through $1 \mathrm{kA}$. This beam is injected into the buncher. It may be a travelling wave tube (TWT) working in the amplification mode. It was shown in [8] that a high degree of bunching can be rather easily achieved in the TWT at the distance of $\sim 1 \mathrm{~m}$. Then the electron bunches continue moving in the LIA in accompaniment of the enhanced in the TWT microwave and simultaneously are accelerated in the LIA electric field. The microwave power extraction from the driver occurs only in the transition chambers. The system attains the steady state at first few tens of meters where the bunch energy increases up to the level of $\sim 10 \mathrm{MeV}$. Then the section of quasi-stationary microwave generation begins where the total power, that the accelerating field inserts into the beam, transforms into the microwave power. The scheme has the following merits: 1 . The possibility of providing the microwave phase and amplitude stability. The phase stability can be obtained at the expense of quasi-continuity of the system. It means that only mechanical tolerances will be of consequence; 2 . Due to the bunched beam acceleration it is not necessary to have a high $(\sim 10 \mathrm{MeV})$ energy of the initial electron beam bunching.

\section{SIMULATION RESULTS}

We have three characteristic regions of the driver: 1) the bunching region with using of TWT and without acceleration, 2) transition region with a beam acceleration and 3) quasi-stationary beam propagation region. As it was shown in [9], one can obtain the electron beam bunching in a TWT at a rather short length $\sim 50 \mathrm{~cm}$ for the following electron beam and $E_{01}$ type wave parameters:

*Work is supported by the Russian Foundation for Basic Research, grants 97-02-16643 and 98-02-17685. 
electron beam energy

electron current inside TWT

$\sim 2.2 \mathrm{MeV}\left(\gamma_{0} \sim 5.31\right)$

$I_{b} \sim 500 \mathrm{~A}$

$\sim 0.5 \mathrm{~cm}$

electron beam radius

$\begin{array}{lr}\text { microwave frequency } f_{0}=17 \cdot 10^{9} \mathrm{~Hz}(\lambda \sim 1.76 \mathrm{~cm}) \\ \text { initial microwave power in TWT } & 10 \mathrm{~kW}\end{array}$

output microwave power in TWT

at the acceleration region entrance

13 MW.

Without accompanying microwave, the debunching process will immediately occur at the distance of about few tens of centimeters. The simulation showed that the electron bunches can be transported at the distance $\sim 10$ $\mathrm{m}$ if the bunch movement is accompanied by the microwave amplified in the TWT. The most encouraging situation is when the moving after the TWT output bunches are accompanied simultaneously by the amplified microwave and are accelerated in the external electric field. inside a corrugated waveguide. The waveguide must be transparent for a pulse accelerating field and a good screen for the microwave.

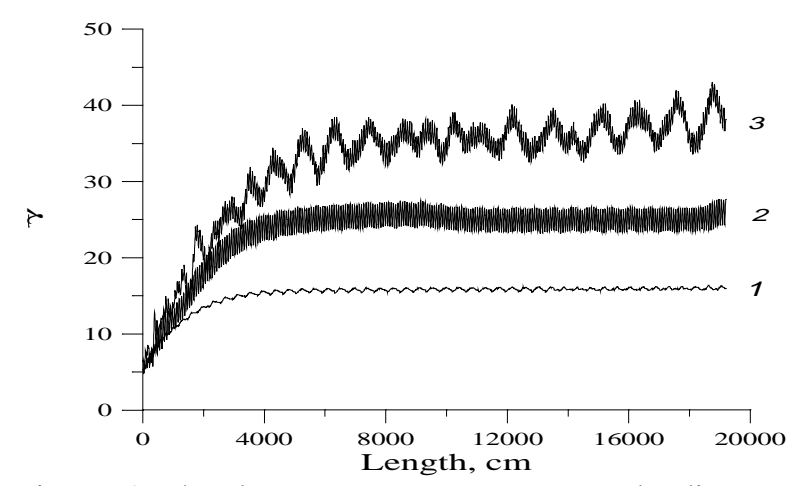

Figure: 1 The electron mean energy versus the distance for: 1 - continuously distributed driver parameters; 2 discrete periodic cells, 3 -discrete cells with narrow accelerating gaps.

Our calculations showed that the bunching process in the TWT must be interrupted some earlier the moment when the bunching parameter $B$ attains its maximum value. Then we have no particle losses in our further calculations in the driver. Fig. 1 shows the calculated dependence of the bunch mean energy $\bar{\gamma}$ on the distance $(z=0$ corresponds to the TWT output). The dependencies of the microwave power and the bunching parameter $B$ on the distance are depicted in the Fig. 2 and Fig. 3. As one can see from these figures, the steady state of the system is achieved at the distance $\sim 40 \mathrm{~m}$. The extracted microwave power in the steady state is equal to $\sim 330 \mathrm{MW}$. The mean steady state bunch energy $\sim 7.5 \mathrm{MeV}$. And at last we have rather high bunching parameter $B \cong 0.9$ in the steady state. The electron phase picture (see Fig. 4) shows that an initial bunch breaks down on two main bunches being at $\Delta \psi \cong 2 \pi$ one from another.

The power put into the electron beam by external electric field is equal to $\sim 500 \mathrm{MW} / \mathrm{m}$. And it trans-

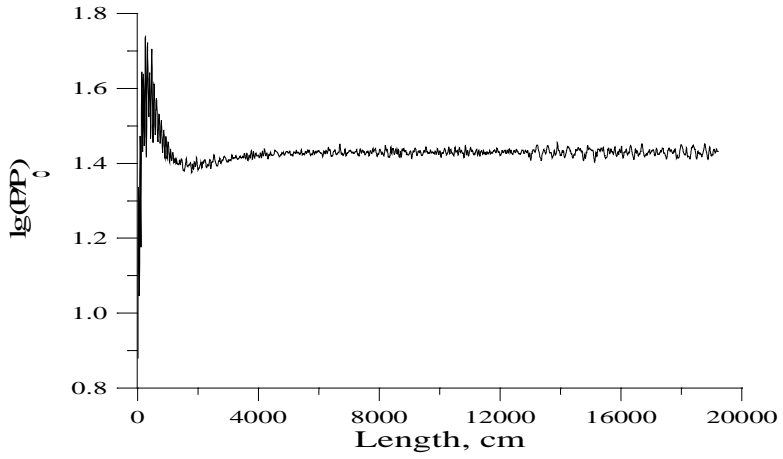

Figure: 2 The microwave power versus the distance for continuously distributed driver parameters.

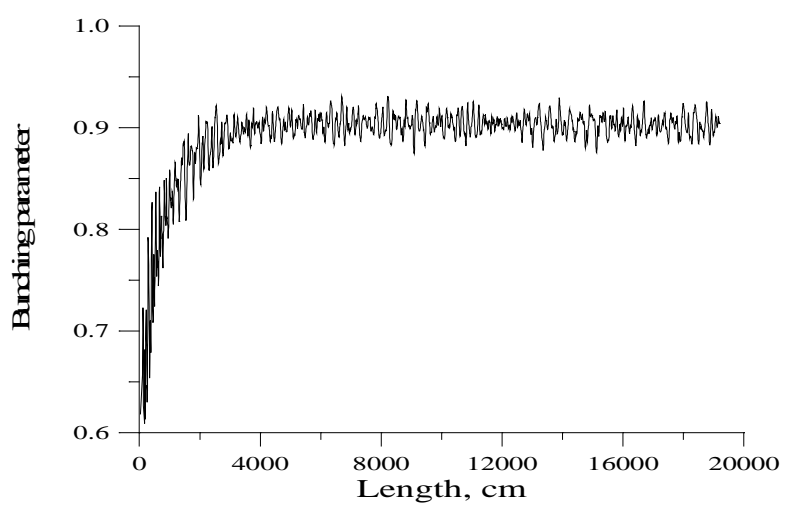

Figure: 3 The bunching parameter versus the distance for continuously distributed driver parameters.

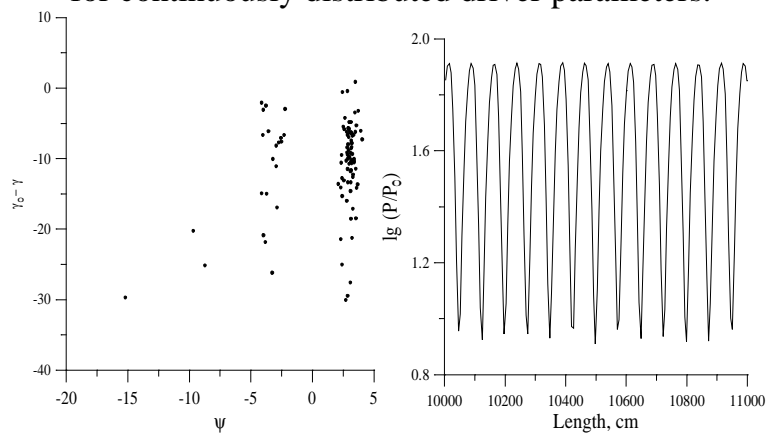

Figures: 4 and 5 The typical electron phase space picture at the distance of $100 \mathrm{~m}$ for continuously distributed driver parameters and the microwave power versus the distance from $100 \mathrm{~m}$ through $110 \mathrm{~m}$ for discrete periodic cells.

forms into the microwave power in the steady state. The stability of the steady state solution with respect to the possible error in the electron beam current $I_{b}$ was also investigated. The calculations showed that we still obtained the steady state solution up to error values $\delta I / I \approx \pm 10 \%$.

A real LIA always consists of separate induction sections partitioned by transition chambers, so that it has a discrete structure. The second set of our calculations was performed for the realistic induction section parameters. As a result, the optimal lengths of 
the induction sections $\left(l_{s}=50 \mathrm{~cm}\right)$ and transition chambers $\left(l_{t}=25 \mathrm{~cm}\right)$ were found. The microwave extraction was switched on only in the transition chambers. And the electric field $E_{a}$ was equal to 1.5 $\mathrm{MV} / \mathrm{m}$ inside the accelerating sections.

The dependence of the bunch mean energy on the distance is depicted in the Fig. 1 (curve 2). One can see a short range of dependence of the microwave power along the distance of $10 \mathrm{~m}$ (from $z=100 \mathrm{~m}$ through $z=110 \mathrm{~m}$ ) in Fig. 5.

In the third set of calculations for the same lengths of the accelerating sections and the transition chambers the only difference was the following: accelerating potential of every section $(750 \mathrm{kV})$ was concentrated only on the gap $7.5 \mathrm{~cm}$ long located at the beginning of every section to make more simple waveguide in this case. The dependence of the bunch mean energy on the distance is depicted in the Fig. 1 (curve 3). The steady state occurs also when the system has the discrete structure. The bunching parameter $B$ stays also high along the driver $(B \cong 0.9)$. The microwave power amplitude stays rather stable.

\section{TEST FACILITY}

Series of test experiments are prepared at JINR to study the scheme mentioned above. The scheme of the experimental setup based on the existing LIA-3000 facility is shown in Fig. 6. It consists of the injector and two reaccelerating sections. At the injector output the electron beam energy is about $800 \mathrm{keV}$ and the current is equal to $200 \mathrm{~A}$. The input microwave power is about of $10 \mathrm{~kW}$ with the wave length of $8 \mathrm{~mm}$. Each reaccelerating section is $130 \mathrm{~cm}$ long and gives the energy gain of $360 \mathrm{keV}$. The microwave extraction and reacceleration voltage in this scheme are both located in the narrow gaps between accelerating sections each few centimetres long.

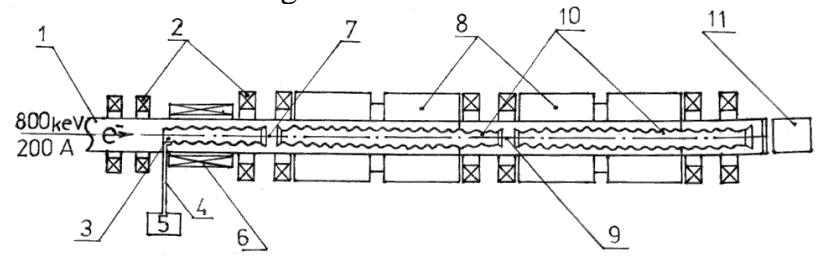

Figure 6: The experimental setup scheme on the base of LIA-3000: 1) injector exit; 2) magnetic field coils; 3) travelling wave tube; 4) waveguide; 5) magnetron; 6) magnetic solenoid; 7) first accelerating gap; 8) linear induction accelerating sections; 9) second accelerating gap;10) corrugated waveguide; 11) diagnostic chamber.

The fulfilled simulation of the beam propagation, acceleration and microwave extraction for the real LIA-
3000 parameters gives that the extracted microwave power should amount by approximately 10-15 MW per gap. The obtained spatial evolution of the mean bunch energy is presented in Fig. 7. Two rapid increases in the energy exactly correspond to the accelerating gaps. From the figures one can see that the travelling electron beam maintain rather high bunching parameter, while the beam energy rises from 0.8 to $1.3 \mathrm{MeV}$.

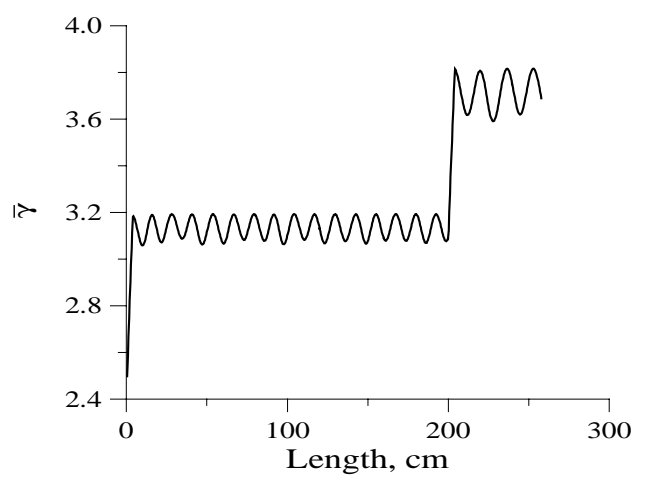

Figure 7: The electron mean energy versus the distance for LIA-3000 parameters. Point $z=0$ corresponds to the beginning of the first accelerating gap in Fig. 6.

The development of the electron bunch diagnostics was carried out in the beginning of our designed experiments on the first facility base. The electron bunch dimensions were measured at the output of the FEL oscillator [9] with the help of the streak camera (Fig. 8). Sweep duration is equal to $700 \mathrm{ps}(50 \mathrm{ps} / \mathrm{mm})$.

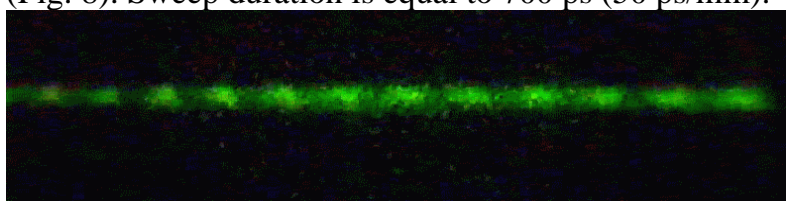

Figure 8: Temporal profile of the bunched electron beam.

\section{REFERENCES}

[1] A.M. Sessler, Proc. Workshop on the Laser Acceleration of Particles, eds. C. Yoshi and T. Katsouleas, AIP Conf. Proc. 91 (1982), p. 154.

[2] A.V. Elzhov, V.I. Kazacha, E. A. Perelstein, Proceedings of XV International Seninar on Charged Particle Linear Accelerators, Kharkov (the Ukraine), v. II (1997), p. 129.

[3] A.M. Sessler et al., Nucl. Instr. Meth., A306, (1991), p. 592.

[4] T. Houck et al., IEEE Trans. on Plasma Science, vol.24, 3 (1996), p. 938.

[5] G.G. Denisov et al., Nucl. Instr. Meth., A358 (1995), p. 528.

[6] T.L. Houck and G.A. Westenskow, Preprint UCRL-JC-119021, LNLL, Livermore, California, USA, 1994.

[7] W. Schnell, CERN-LEP-RF/86-27 (1986); CERN-LEP-RF/8859 (1988).

[8] N.S.Ginzburg et al., in: Relativistic High-Frequency Electronics, v. 5 (IAP AS USSR, Gorky, 1988), p. 37.

[9] E.A. Perelstein et al, JINR Preprint E9-97-5, Dubna, 1997. 